\title{
The outcomes of epiretinal membrane peeling in patients with foveal herniation
}

CrossMark

\author{
Abdullah Ozkaya*, Gurkan Erdogan and Gokhan Demir
}

\begin{abstract}
Background: To evaluate the surgical outcomes of vitrectomy in patients with foveal herniation associated with epiretinal membrane (ERM).

Methods: This was a retrospective case series. The patients who had a foveal herniation associated with ERM, underwent vitrectomy, and had a minimum follow-up period of 12 months were included. The visual and anatomical outcomes were assessed during the first 12 months of follow-up and at the last follow-up visit. The main outcome measure was the reorganization pattern of foveal pit at month 12 .

Results: Eleven eyes of 11 patients were included. The mean postoperative follow-up period was $14.8 \pm 2.6$ months. The foveal contour was completely restored in 5 eyes (45.5\%), partially restored in 5 eyes (45.5\%), and was not restored in 1 eye $(9.1 \%)$ at postoperative month 12 follow-up visit. The mean preoperative best corrected visual acuity was $0.61 \pm 0.16$ LogMAR and increased to $0.49 \pm 0.16$ LogMAR at month $12(p<0.0001)$. The mean preoperative central retinal thickness was $476 \pm 128$ micrometers and decreased to $302 \pm 70$ micrometers at month $12(p<0.0001)$.
\end{abstract}

Conclusion: The foveal contour was restored in $45.5 \%$ of the eyes and visual acuity was significantly increased by a mean of 1.2 LogMAR lines at month 12 in patients with foveal herniation associated with ERM.

Keywords: Epiretinal membrane, Foveal herniation, Vitrectomy

\section{Background}

Epiretinal membrane (ERM) is the most frequent vitreomacular surface disorder [1-4]. Surgical treatment is usually required if it causes metamorphopsia and visual deterioration, and consists of vitrectomy and ERM peeling [4-6]. After the introduction of optical coherence tomography (OCT), different ERM subtypes have been described [7-9]. The ERMs have been classified into diffuse, cystoid macular edema, vitreomacular traction, and pseudolamellar hole subtypes [7]. An interesting and new subtype of ERM was described by Ozdemir and Karacorlu in 2017 as 'foveal herniation" [10]. This subtype was previously classified as "outer retinal inward projection and inner retinal thickening" by Hwang et al. [8]. However, there is little data with regards to the surgical outcomes of this specific subtype. Therefore, we aimed to

*Correspondence: abdozkaya@gmail.com

Beyoglu Eye Training and Research Hospital, Bereketzade Cami Sok., 34421 Beyoglu, Istanbul, Turkey investigate the surgical outcomes of the ERM patients with foveal herniation.

\section{Methods}

The records of the ERM patients who underwent 23-gauge vitrectomy between January 2016 and February 2017 were reviewed for this retrospective case series. A total of 11 patients showed foveal herniation and were included in the study. A written consent was obtained from all of the patients preoperatively. Local review board approval was obtained and the study was in adherence with the tenets of the Declaration of Helsinki.

The patients with a diagnosis of idiopathic ERM and who showed foveal herniation and had a minimum postoperative follow-up period of 12 months were included (Fig. 1). The patients who had a concomitant vascular retinal disease were not included. Age, gender, best corrected visual acuity (BCVA), central retinal thickness (CRT), and complications were collected retrospectively from the patients' medical records. The patients 
Pre-operative examinations, 11 eyes BCVA, optical coherence tomography based CRT

Pars plana vitrectomy and epiretinal membrane peeling, 11 eyes

Post-operative visits at month $1,3,6$, and 12,11 eyes $B C V A$, optical coherence tomografi based CRT, and formation of foveal pit.

Fig. 1 The flow chart of the study method

underwent a standard ocular examination which included BCVA measurement via a projection chart in decimals, biomicroscopy (Heig Streit AG, Koning Switzerland), intraocular pressure measurement via applanation tonometer (Heig Streit AG, Koning Swizerland), and fundus examination via 90 diopter lens (Volk Optical Inc, Mentor, Ohio, USA). Optical coherence tomography imaging was performed (Spectralis; Heidelberg Engineering, Heidelberg, Germany) preoperatively. The examinations were repeated at post-operative day 1 , week 1 , and months $1,3,6$, and 12 . Central retinal thickness, defined as the mean thickness of the neurosensory retina in a central $1 \mathrm{~mm}$ diameter area, was computed using OCT mapping software generated by the device. The diagnosis of foveal herniation was made as previously described [10]. The patients who showed a protruded retinal tissue through the ERM were diagnosed with foveal herniation (Fig. 2). At postoperative month 12 the pattern of the formation of foveal contour was divided into 3 groups. The foveal pit was accepted as fully restored if there was a well-formed foveal pit on the OCT scan, it was accepted as partially restored if a pit was detected but was not fully formed, and was accepted as not restored if a pit was not detected.

\section{Surgical technique}

All of the operations were performed by two experienced vitreoretinal surgeons (A.O. and G.E.). The patients underwent 23-gauge vitrectomy with the Constellation system (Alcon Surgical, Ft. Worth, TX). A wide-field viewing system was used. Patients with a significant cataract underwent simultaneous phacoemulsification in the same session with the vitrectomy procedure.

After preparing the three standard ports for vitrectomy, core vitrectomy was performed and posterior vitreous was checked for the presence of a posterior vitreous detachment (PVD) with the help of triamcinolone and PVD was induced if was not present. Then a limited posterior vitrectomy was performed as previously described [11]. Epiretinal membrane was stained with trypan-blue

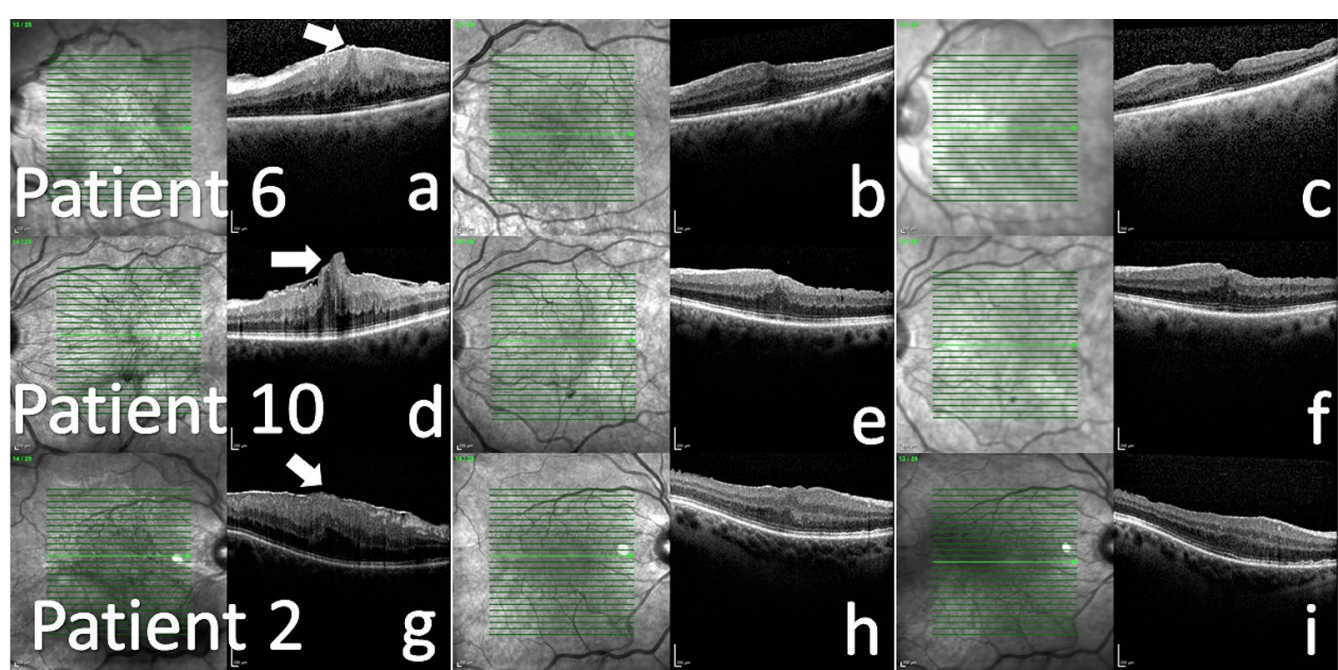

Fig. 2 The examples of eyes with fully, partially or not restored foveal contour, a-c the optical coherence tomography scans of patient 6 at baseline, postoperative month 3 and 12, who showed fully restored foveal pit at month 12 . White arrow indicated the tiny foveal herniation at baseline, $\mathbf{d}-\mathbf{f}$ the optical coherence tomography scans of patient 10 at baseline, postoperative month 3 and 12, who showed partially restored foveal pit at month 12. White arrow indicated the prominent foveal herniation at baseline, $\mathbf{g}-\mathbf{i}$ the optical coherence tomography scans of patient 2 at baseline, postoperative month 3 and 12, in whom foveal pit was not restored at month 12. White arrow indicated the tiny foveal herniation at baseline 
(0.06\%, VisionBlue, DORC, Netherlands) and peeled via a 23-gauge microforceps. After peeling the ERM, the continuity of the internal limiting membrane (ILM) was checked with the help of brilliant blue G (Fluoron Gmbh, NEU-Ulm, Germany) and ILM was also peeled if it was suspiciously or clearly disrupted. At least two-disc diameters of ERM was peeled (Fig. 3). Retina was examined with indentation at the end of the surgery to detect new or previous retinal breaks and barrier laser photocoagulation was performed when required. Tamponade choice was not based on any specific rules and positioning was not suggested according to the used tamponade.

Primary outcome measure was the formation pattern of foveal contour at month 12 after the surgery. Secondary outcomes were the change in BCVA and CRT.

\section{Statistical analysis}

Statistical Package for the Social Sciences (SPSS) software (version 21.0) were used for statistical analyses. Visual acuity was converted to the logarithm of the minimum angle of resolution (LogMAR) for statistical analysis. The data was analyzed in terms of normal distribution with Shapiro-Wilk test. As the distribution of the data was normal, visual acuity and the CRT values between baseline and the other time points were assessed with repeated measures test. Categorical variables were compared using Chi-square test. A $p$ value $<0.05$ was considered statistically significant.

\section{Results}

Eleven eyes of 11 patients were included in the study. The mean age was $65.0 \pm 5.5$ years (range $61-80$ years). Eight patients $(72.7 \%)$ were female and $3(27.3 \%)$ were male. The mean postoperative follow-up period was

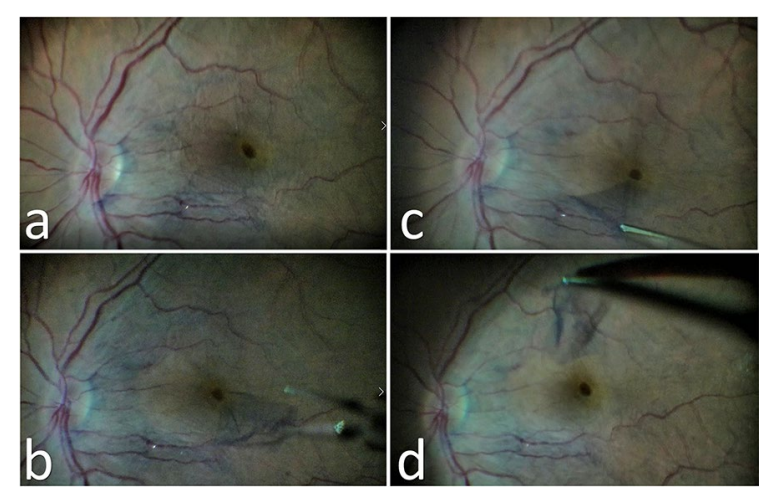

Fig. 3 The peroperative screenshots of a patient, a the epiretinal membrane was stained with trypan-blue and the foveal herniation is detected like a pseudohole, $\mathbf{b}$, c epiretinal membrane is being peeled and it is clearly seen that it has an operculum at the center, $\mathbf{d}$ the end of the peeling foveal herniation is still detected like a pseudohole
$14.8 \pm 2.6$ months (range 12-20 months). Six eyes (54.4\%) were phakic and $5(45.5 \%)$ were pseudophakic preoperatively. Two of the six phakic eyes (33.3\%) underwent cataract surgery with phacoemulsification at the time of the vitreoretinal surgery. The general characteristics of the patients were listed in Table 1.

The foveal contour was fully restored in 5 eyes (45.5\%), partially restored in 5 eyes (45.5\%), and was not restored in 1 eye $(9.1 \%)$ at postoperative month 12 follow-up visit (Fig. 2).

The mean preoperative BCVA was $0.61 \pm 0.16$ LogMAR (range 0.4-1.0 LogMAR). The mean BCVA at postoperative months $1,3,6,12$ and the last follow-up was $0.49 \pm 0.12$ (range $0.22-0.7$ ), $0.47 \pm 0.12$ (range 0.22-0.7), $0.54 \pm 0.22$ (range $0.1-1.0$ ), $0.49 \pm 0.16$ (range 0.15-0.7), and $0.49 \pm 0.17$ LogMAR (range 0.15-0.7), respectively $(p<0.0001$ for all time points versus preoperative BCVA) (Fig. 4). Two eyes (18.1\%) gained $\geq 3$ lines of vision, the remaining 9 eyes $(81.9 \%)$ remained stable (gained $<3$ lines, did not change, or lost $<3$ lines).

The mean preoperative CRT was $476 \pm 128$ micrometers (range 268-660). The mean CRT at postoperative months 1, 3, 6, 12 and the last follow-up was $362 \pm 44$ (range 274-428), 317 \pm 80 (range 120-385), $304 \pm 79$ (range 140-372), $302 \pm 70$ (range 170-388), and $303 \pm 61$ micrometers (range $180-370)$, respectively $(p<0.0001$ for all time points versus preoperative BCVA) (Fig. 5).

Solely ERM was peeled in 3 eyes (27.3\%), and both ERM and ILM were peeled in 8 eyes $(72.7 \%)$. None of the eyes showed ERM recurrence during the follow-up period. The mean BCVA was $0.54 \pm 0.15$ LogMAR (range $0.4-0.7$ ) and $0.64 \pm 0.16 \operatorname{LogMAR}$ (range 0.52-1.0) in patients in whom only ERM and both ERM and ILM were peeled, respectively $(p=0.3)$. The mean visual acuity was increased by 1.5 and 1.1 lines in only ERM and both ERM and ILM peeled eyes, respectively $(p=0.8)$. No tamponade was used in $5(45.5 \%)$, air was used in 3 (27.3\%), sulphur hexafluoride was used in $1(9.1 \%)$, and perfluoropropane was used in 2 eyes (18.2\%).

Three of the 4 remaining phakic eyes $(75 \%)$ required cataract surgery during the follow-up period. No significant peroperative complications occurred during the surgeries. Only one eye had a previously treated peripheral retinal tear, and at postoperative month 2 this eye progressed to retinal detachment. This eye was operated again and had an attached retina at the last follow-up visit.

\section{Discussion}

We reviewed the outcomes of vitrectomy in 11 patients with ERM associated with foveal herniation. Foveal contour was completely restored in nearly half of the patients and the visual acuity increased significantly in 


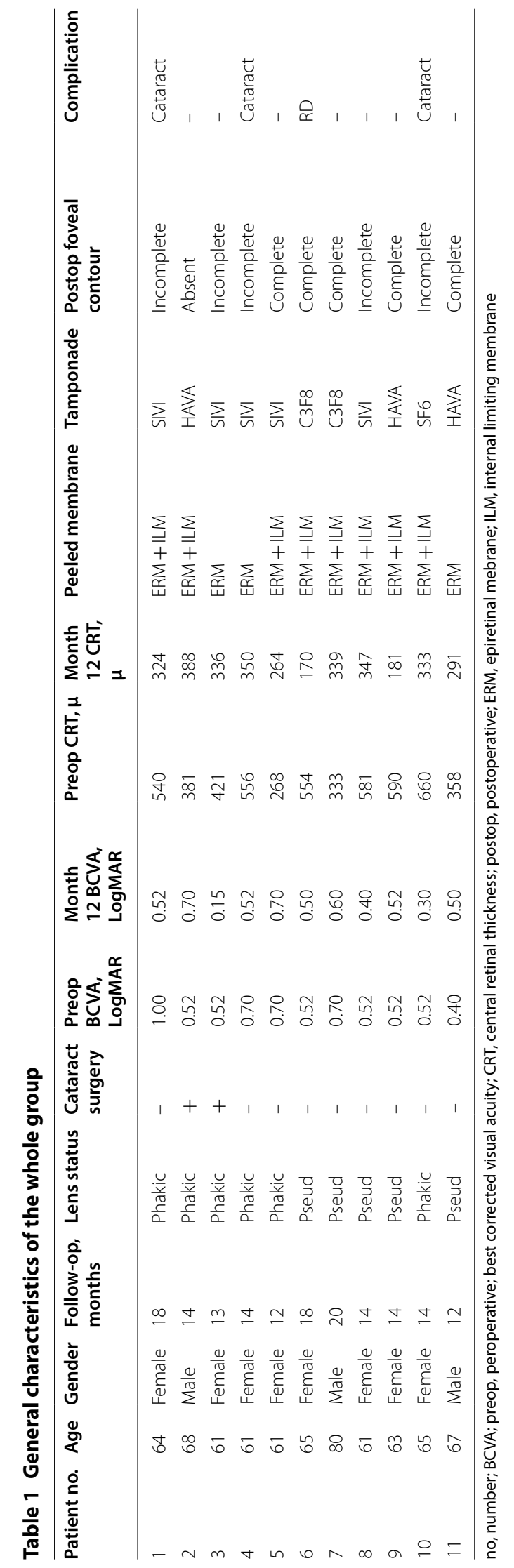




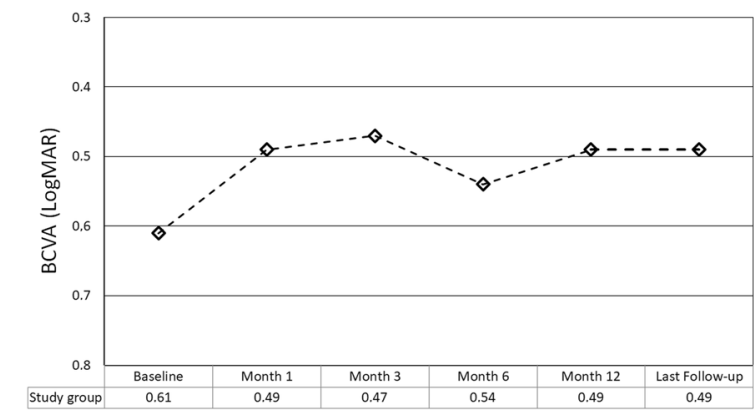

Fig. 4 The change in best corrected visual acuity throughout the follow-up period (BCVA, best corrected visual acuity)

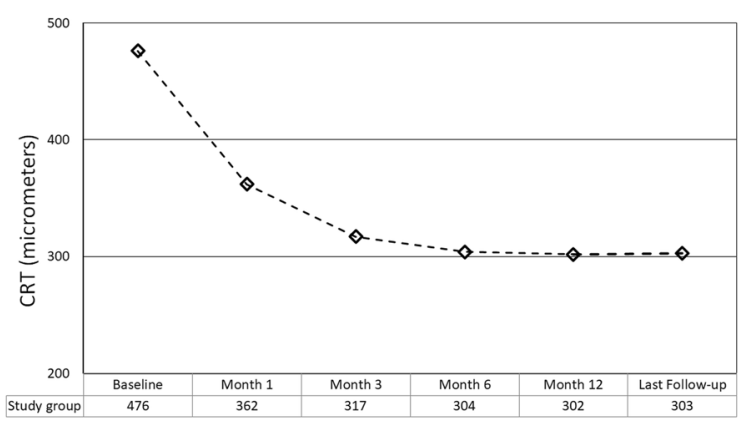

Fig. 5 The change in central retinal thickness throughout the follow-up period (CRT, central retinal thickness)

two patients at month 12. Also, the mean CRT decreased significantly at the postoperative period throughout the first year. The visual and anatomical outcomes of this study were similar to previous studies in terms of vitrectomy outcome in ERM patients $[4-7,11]$ We documented an increase of 1.2 LogMAR lines in visual acuity at month 12 in this study. Kinoshita et al. [7] evaluated the time course of changes in metamorphopsia, visual acuity and OCT parameters after ERM surgery in their study. The visual acuity was found to be increased from 0.38 LogMAR to 0.09 LogMAR at month 12 and $67.3 \%$ eyes showed $\geq 2$ lines of visual increase. In a previous study by our group, we evaluated the outcomes of subtotal vitrectomy in patients with ERM and macular hole [11]. After a follow-up period of 12 months the BCVA increased from 0.65 LogMAR to 0.56 LogMAR. The change in BCVA was evaluated in a meta-analysis by Azuma et al. [5]. The change in mean BCVA ranged from -0.05 LogMAR lines up to +0.46 LogMAR lines in ERM + ILM peeled patients and between -0.13 and +0.42 LogMAR lines in only ERM peeled eyes. Internal limiting membrane peeling is an important adjuvant to ERM peeling in patients with ERM and has been to show to decrease the recurrence rate [5]. Azuma et al.
[5] also evaluated the recurrence rate of ERMs in a total of 1201 eyes in their valuable meta-analysis. The rate of ERM recurrence was reported to be $2.0 \%$ (13/645 eyes) in ERM + ILM peeling group and $11.0 \%$ (66/600 eyes) in only ERM peeling group and the change was found to be statistically significant between the two groups. We used a standard technique of observing the integrity of ILM after the peeling of ERM. The integrity and continuity of ILM was checked with the help of brilliant blue G in all of our cases and we peeled the ILM only in the event of disruption or suspicion of disruption. In this study most of the eyes showed disruption of ILM after the peeling of ERM, and we peeled the ILM in 8 of 11 included eyes. None of our included patients showed recurrence of ERM during the follow-up period. This may be because of small sample size and also secondary to the high rate of ILM peeled eyes. We also evaluated the visual change at month 12 between the subgroups of patients in whom only ERM (1.6 lines) was peeled and both ERM and ILM (1.1 lines) were peeled, and did not find statistically significant difference between the subgroups.

Several types of epiretinal membranes were defined in previous studies [3, 4, 7-9, 12, 13]. Hwang et al. [8] were the first authors to define the later so called "foveal herniation" subtype as "outer retinal inward projection and inner retinal thickening. In that study, they mainly divided the ERMs into two main groups as fovea attaching (1) and fovea sparing groups (2) and the former one had 3 groups while the latter had 2 . The foveal herniation group was defined as subtype 1B. They evaluated the macular function via multifocal electroretinography (mfERG) and reported that the eyes in group 2 had better foveal function than the eyes in group 1 in regards to mfERG outcomes. The study demonstrated further data concerning inter-group changes and correlation with the fellow eyes of the patients; however, this was a crosssectional study and did not give longitudinal data about these patients. Kinoshita et al. [7] divided the ERMs into 4 groups as diffuse, cystoid macular edema, pseudolamellar hole, and vitreomacular traction (VMT) subtypes. The subtype of the ERM was evaluated as a predictive factor for the surgical outcome in the study, but there was not any further data and whether the included eyes showed a lesion like foveal herniation or not were not reported. They concluded that the VMT group showed better visual outcomes and also higher recurrence than the other groups. Although several authors also made different classifications according to morphology of ERMs on OCT, Ozdemir and Karacorlu were the first authors who used the term "ERM with foveal herniation" [10]. They reported a 65-year-old woman who had an ERM with foveal herniation in the left eye. The authors described the OCT appearance as the herniation of the superficial 
layers of the retina towards the vitreous through an opening in the center of the ERM.

Although it is a well-known fact that the formation of ERMs are related with PVD [14-16], data concerning the possible mechanisms of the formation of a foveal herniation and surgical outcomes are scarce in the literature. At the first stages of PVD, vitreous is found to be detached from the fovea, but attached to elsewhere around it [17, 18]. Perhaps, while posterior vitreous detaches from the retinal surface, the cortical vitreous might completely detach from the foveola but remain attached firmly around the fovea during these early stages. This may lead to the vitreous remnants on the retinal surface during the detachment of the posterior vitreous from the perifoveal area, a phenomenon previously described as vitreoschisis. If these remnants progress to ERM and contractile cells get involved into this process, this new ERM may create a centrifugal contraction and cause inner retinal layers to protrude from the central operculum to form a foveal herniation. Although this is-at least for now-a little bit hypothetical, it may be one of the possible mechanisms underlying the formation of foveal herniation.

The main limitations of this study were the small study population and its retrospective design. However, foveal herniation is a special and rare subtype of ERM. To the best of our knowledge, we evaluated for the first time the surgical outcomes of foveal herniation in 11 patients during a follow-up time of 12 months.

As a conclusion, the surgical outcomes were satisfactory and similar to the previous ERM series. Nearly half of the included eyes showed a fully formed foveal contour at month 12. Visual and anatomical outcomes were also acceptable and similar to the previous studies. Further studies with a longer follow-up period are required to evaluate the clinical significance of this specific subtype of ERM. Similarly, the true incidence of foveal herniation and its surgical outcomes are yet to be determined in larger series.

\section{Abbreviations}

ERM: epiretinal membrane; BCVA: best corrected visual acuity; CRT: central retinal thickness; OCT: optical coherence tomography; PVD: posterior vitreous detachment; ILM: internal limiting membrane; LogMAR: logarithm of the minimum angle of resolution; VMT: vitreomacular traction.

\begin{abstract}
Authors' contributions
Involved in conception and design of the study (AO, GE, GD); acquisition of data (AO, GD); conceptualization of the manuscript and review and synthesis of the literature; (AO, GE, GD); and analysis and interpretation of data (AO); drafting the manuscript $(\mathrm{AO})$; critical review and revision of the manuscript $(A O, G E, G D)$. All authors have read and approved the final manuscript.
\end{abstract}

\section{Acknowledgements}

The authors thank to Dr. Hande Mefkure Ozkaya for her to review the manuscript for English editing.

\section{Competing interests}

The authors declare that they have no competing interest.

Availability of data and materials

Not applicable.

\section{Consent for publication}

The manuscript does not contain any data or image that reveals subject details.

\section{Ethical standards}

The study was in adherence to the Declaration of Helsinki, and consent forms were obtained from all of the patients before each treatment applied in the study. Local review board approval was obtained.

\section{Funding}

This retrospective study was not supported by any company. None of the authors has financial or proprietary interests in any material or method mentioned. This data has not been previously published.

\section{Publisher's Note}

Springer Nature remains neutral with regard to jurisdictional claims in published maps and institutional affiliations.

Received: 18 August 2018 Accepted: 24 October 2018

Published online: 07 November 2018

\section{References}

1. Bu SC, Kuijer R, Li XR, et al. Idiopathic epiretinal membrane. Retina. 2014;34:2317-35

2. Dupas B, Tadayoni R, Gaudric A. Epiretinal membranes. J Fr Ophtalmol. 2015;38:861-75 (in French).

3. Stevenson W, Prospero Ponce CM, Agarwal DR, et al. Epiretinal membrane: optical coherence tomography-based diagnosis and classification. Clin Ophthalmol. 2016;10:527-34.

4. Miguel Al, Legris A. Prognostic factors of epiretinal membranes: a systematic review. J Fr Ophtalmol. 2017;40:61-79.

5. Azuma K, Ueta T, Eguchi S, Aihara M. Effects of internal limiting membrane peeling combined with removal of idiopathic epiretinal membrane: a systematic review of literature and meta-analysis. Retina. 2017;37:1813-9.

6. Kinoshita T, Imaizumi H, Okushiba U, et al. Time course of changes in metamorphopsia, visual acuity, and OCT parameters after successful epiretinal membrane surgery. Invest Ophthalmol Vis Sci. 2012;53:3592-7.

7. Kinoshita T, Kovacs KD, Wagley S, Arroyo JG. Morphologic differences in epiretinal membranes on ocular coherence tomography as a predictive factor for surgical outcome. Retina. 2011;31:1692-8.

8. Hwang JU, Sohn J, Moon BG, et al. Assessment of macular function for idiopathic epiretinal membranes classified by spectral-domain optical coherence tomography. Invest Ophthalmol Vis Sci. 2012;53:3562-9.

9. Aydın R, Karahan E, Kaya M, et al. Evaluation of inner segment/outer segment junctions in different types of epiretinal membranes. Arq Bras Oftalmol. 2016;79:319-22

10. Ozdemir H, Karacorlu M. Epiretinal membrane with foveal herniation. Retina. 2017;37:e71-2.

11. Ozkaya A, Erdogan G, Tulu B, Tarakcioglu HN. The outcomes of subtotal vitrectomy in macular surgeries: a single surgeon case series. Int Ophthalmol. 2018. https://doi.org/10.1007/s10792-018-0850-1.

12. Liu X, Ling $Y$, Huang J, Zheng $X$. Optic coherence tomography of idiopathic macular epiretinal membranes. Yan Ke Xue Bao. 2002;18:14-9.

13. Zampedri E, Romanelli F, Semeraro F, et al. Spectral-domain optical coherence tomography findings in idiopathic lamellar macular hole. Graefes Arch Clin Exp Ophthalmol. 2017;255:699-707.

14. Sidd RJ, Fine SL, Owens SL, Patz A. Idiopathic preretinal gliosis. Am J Ophthalmol. 1982:94:44-8. 
15. Ota A, Tanaka Y, Toyoda F, et al. Relationship between variations in posterior vitreous detachment and visual prognosis in idiopathic epiretinal membranes. Clin Ophthalmol. 2015;10:7-11.

16. Sebag J. Anomalous posterior vitreous detachment: a unifying concept in vitreo-retinal disease. Graefes Arch Clin Exp Ophthalmol. 2004;242:690-8.

17. Mirza RG, Johnson MW, Jampol LM. Optical coherence tomography use in evaluation of the vitreoretinal interface: a review. Surv Ophthalmol. 2007;52:397-421.
18. Tsukahara M, Mori K, Gehlbach PL, Mori K. Posterior vitreous detachment as observed by wide-angle OCT imaging. Ophthalmology. 2018. https:// doi.org/10.1016/j.ophtha.2018.02.039.
Ready to submit your research? Choose BMC and benefit from:

- fast, convenient online submission

- thorough peer review by experienced researchers in your field

- rapid publication on acceptance

- support for research data, including large and complex data types

- gold Open Access which fosters wider collaboration and increased citations

- maximum visibility for your research: over $100 \mathrm{M}$ website views per year

At BMC, research is always in progress.

Learn more biomedcentral.com/submissions 